\title{
Differential risk of disease progression between isolated anastomotic ulcers and mild ileal recurrence after ileocolonic resection in patients with Crohn's disease
}

\author{
Jacob E. Ollech, MD ${ }^{1,{ }^{*},}$ Maya Aharoni-Golan, MD ${ }^{1,{ }^{*}}$, Roni Weisshof, MD ${ }^{1}$, Inessa Normatov, \\ MD1 ${ }^{1}$, Abby R. Sapp, MD², Aditya Kalakonda, DO $^{3}$, Amanda Israel, MD ${ }^{1}$, Laura R. Glick, BA ${ }^{1}$, \\ Theodore Karrison, PhD ${ }^{4}$, Sushila R. Dalal, MD1 , Atsushi Sakuraba, MD1, Russell D. Cohen, \\ MD', David T. Rubin, MD1', Joel Pekow, MD1 \\ ${ }^{1}$ Inflammatory Bowel Disease Center, University of Chicago Medicine, Chicago, Illinois, USA \\ ${ }^{2}$ Department of Gastroenterology, Franciscan Alliance, Hammond, Indiana, USA \\ ${ }^{3}$ Baystate Medical Center, University of Massachusetts, Springfield, Massachusetts, USA \\ ${ }^{4}$ Department of Public Health Sciences, Biostatistics Lab, University of Chicago, Chicago, Illinois, \\ USA.
}

\section{Abstract}

Background and Aims: It is standard of care to perform ileocolonoscopy within a year of ileocolonic resection for Crohn's disease (CD) and to guide management decisions based on the Rutgeert score (RS). The modified RS subdivides i2 into lesions confined to the anastomosis (i2a) or $>5$ aphthous lesions in the neoterminal ileum (i2b). There is uncertainty, however, if i2a lesions incur an increased risk of disease recurrence. The primary aim of this study was to compare the rates of endoscopic progression between $\mathrm{i} 2 \mathrm{a}$ and $\mathrm{i} 2 \mathrm{~b}$ when compared with $\mathrm{i} 0 \mathrm{i} \mathrm{i} 1$.

Methods: This was a retrospective, single-center study including patients with CD who had an ileocolonoscopy $\leq 2$ months after ileocolonic resection with primary anastomosis and who had >1 year of documented clinical follow-up after the index endoscopic evaluation. All consecutive eligible patients between 2004 and 2014 were included in the study. Demographic, disease, and treatment data were collected. Patients with i3 or i4 at index colonoscopy were excluded from further analyses. Outcomes included endoscopic progression and recurrent surgery. For patients with RS of i0 to i2, endoscopic progression was predefined as progression of the RS in subsequent colonoscopies to i3 or i4. Recurrent surgical interventions were defined as re-resection or stricturoplasty of the previous ileocolonic anastomosis.

Results: Two hundred seven CD patients (median age, 36 years [interquartile range, 26-48]) had an ileocolonoscopy $\leq 2$ months after ileocolonic resection. At index colonoscopy, 95 patients (45.9\%) had an RS of i0, 31 (14.9\%) i1, 40 (19.3\%) i2a, 25 (12.1\%) i2b, 10 (4.8\%) i3, and 6 (2.9\%) i4. One hundred ninety-one patients had an RS of i0 to i2 and were included in the

Reprint requests: Jacob E. Ollech MD, University of Chicago Medicine, Inflammatory Bowel Disease Center. MC 4076, Room M421. 5841 S. Maryland Avenue. Chicago, IL 60637.

Drs Ollech and Aharoni-Golan contributed equally to this article.

DISCLOSURE: All other authors disclosed no financial relationships relevant to this publication. 
analyses for recurrent surgery. One hundred forty-nine patients had a second endoscopic evaluation and were included in the analysis for the primary outcome of endoscopic disease progression. Kaplan-Meier analyses were performed and found the hazard ratio (HR) of endoscopic progression to be significantly higher with i $2 b$ lesions when compared with i0 or i1 (HR, 6.22; 95\% confidence interval [CI], 2.38-16.2; $P=.0008$ ). Patients with i2a did not have significantly higher rates of endoscopic progression when compared withi0 or i1 (HR, 2.30; 95\% CI, .80-6.66; $P=.12$ ). Likewise, patients withi2b lesions had higher risk of needing recurrent surgery when compared with i0 or i1 (HR, 3.64; 95\% CI, 1.10-12.1; $P=.034$ ), whereas patients with i2a lesions were not found to have a significantly elevated risk of recurrent surgery (HR, 1.43; $95 \%$ CI, .35-5.77; $P=.62$ ).

Conclusion: Endoscopic lesions limited to the ileocolonic anastomosis (RS i2a) in patients with CD undergoing colonoscopy within 1 year of their resection were not associated with a significantly higher rate of progression to more severe disease, whereas those in the neoileum (RS i2b) were. Prospective studies are needed to confirm these findings. (Gastrointest Endosc 2019;90:269-75.)

Crohn's disease (CD) is characterized by chronic, relapsing intestinal inflammation in a genetically susceptible host. ${ }^{1}$ Medical therapy is aimed at inducing remission and preventing disease relapse and adverse events. Despite the introduction of several cytokine targeted biologics for $\mathrm{CD}$ in the last 20 years, most patients still require surgical intervention, with ileocecal resection the most common procedure. ${ }^{2}$ However, disease recurrence after surgery is common, with $70 \%$ of patients demonstrating lesions in the neoterminal ileum at 1 year after resection. ${ }^{3}$ Several risk factors leading to postoperative disease recurrence have been identified, including smoking, prior intestinal resection, penetrating disease behavior, perianal disease, and extensive bowel disease.,5

The Rutgeert score (RS) is used to assess endoscopic disease recurrence after ileocolonic resection. It has 5 grades of severity (i0-i4), which have been shown to correlate with clinical recurrence ${ }^{6}$ (Table 1). It has become standard of care to assess patients endoscopically within a year of ileocolonic resection to guide management decisions to prevent disease progression. ${ }^{5,7}$ The original RS of i 2 was defined as $>5$ aphthous ulcers in the neoterminal ileum or lesions confined to the anastomosis. ${ }^{6}$ Although it is widely agreed that patients with an $\mathrm{i} 3$ or i4 score have a high risk of clinical recurrence and that patients with an i0 or i1 score have a favorable outcome, ${ }^{4,6}$ there is uncertainty as to whether lesions confined to the anastomosis incur disease recurrence or if these lesions simply imply ischemic changes with minimal or no risk of disease progression. ${ }^{8}$ Thus, a modified RS has been proposed that subdivides $\mathrm{i} 2$ into lesions confined to the anastomosis (i2a) or $>5$ aphthous lesions in the neoterminal ileum (i2b). ${ }^{9}$

A better risk assessment of disease recurrence in patients with RS i2 has significant clinical relevance. Because this is a common clinical tool utilized at the first postoperative colonoscopy to guide therapeutic management, sub-classification of i2 lesions may help to identify patients at greatest risk of recurrence who would most benefit from intensive monitoring and prophylaxis on one hand and may prevent unnecessary treatment in patients with low risk of recurrence on the other. Thus, the aim of this study was to compare the rates 
of endoscopic progression and surgical intervention between $\mathrm{i} 2 \mathrm{a}$ and $\mathrm{i} 2 \mathrm{~b}$ when compared with i0 to i1.

\section{METHODS}

\section{Patient selection}

This was a retrospective, single-center study including patients with $\mathrm{CD}$ who had ileocolonoscopy $₫ 2$ months after ileocolonic resection with primary anastomosis and who had $>1$ year of documented clinical follow-up after the endoscopic evaluation. All consecutive eligible patients between 2004 and 2014 were included in the study. Patients were excluded from further analyses if they had an RS of i3 or i4 at the index colonoscopy. Patients were also excluded if they had intestinal procedures other than open or laparoscopic ileocolonic resection with primary anastomosis, such as small-bowel resection, segmental colonic resection, or a stoma.

\section{Data collection}

Patient demographic, clinical, radiographic, and endoscopic data were collected by a comprehensive review of their electronic medical records. The following baseline characteristics were collected: age at inclusion, age at diagnosis, gender, disease duration, $\mathrm{CD}$ location and behavior based on the Montreal classification, ${ }^{10}$ previous surgery, smoking status, indication for surgery, and bowel anastomosis technique (end-to-end, side-to-side, and end-to-side). All follow-up colonoscopies that reached the anastomosis were recorded.

Colonoscopies after the first postoperative colonoscopy were performed based on the clinician's discretion. The RS was scored by the physician who performed the colonoscopy at the time of endoscopic evaluation. All RSs were reviewed by an investigator who was blinded to previous RS. Validation of the RS was done by reviewing the endoscopic report, which includes a description of the findings and high-definition images of the anastomosis and neoterminal ileum, to attribute the correct modified RS.

Discrepancies between the RS assigned at the time of the endoscopy and the description as well as images of the endoscopic findings were found in 7 cases. All these discrepancies were associated with isolated anastomotic lesions (i2a) and the same 2 endoscopists. In these cases, the colonoscopy reports and pictures correctly described the anastomotic lesion and the normal ileum. However, the endoscopists incorrectly labeled these patients as having RS of $\mathrm{i} 0$ to i1. These findings were reviewed by a third investigator, and the scores were changed to i2a.

Endoscopic progression was defined as progression of the RS in subsequent colonoscopies to $i 3$ or i4. All recurrent surgical interventions after the initial postoperative colonoscopy, defined as re-resection or stricturoplasty of the previous ileocolonic anastomosis, were collected. Exposure to treatments (5-aminosalicylic acid, immunomodulators, steroids, and biologics) before surgery, after surgery but before the first endoscopy, and after each subsequent ileocolono-scopy were also recorded. The study was approved by the institutional ethics review board (IRB17-0064, 1/22/2018). 


\section{Outcomes}

The primary outcome was risk of endoscopic progression for patients with i2 lesions (i2a and i2b) compared with patients with i0 to i1 lesions. The secondary outcome was risk of recurrent surgery for patients with i2 lesions (i2a and i2b) compared with those with i0 to i1 lesions.

\section{Statistical analysis}

Descriptive statistics for demographic and clinical characteristics were median (interquartile range) for continuous variables and frequency distributions for categorical data. KaplanMeier curves were generated for time-to-event data, that is, time from first colonoscopy until endoscopic progression (RS of 3 or 4 ) and time from first colonoscopy until recurrent surgery. Patients who did not reach an RS of 3 or 4 were censored as of the date of the last colonoscopy; patients without recurrent surgery were censored as of the date of last followup. Cox regression models were fit to examine the effects of initial scores on time to endoscopic progression and recurrent surgery as well as the effects of other covariates (age, sex, race, smoking status, previous surgery, disease location and behavior, preoperative treatments, treatment change after the index colonoscopy, and anastomotic technique [endto-end, end-to-side, and side-to-side]). Because of the limited number of events, however, models for time to endoscopic progression included only the RS and 1 covariate at a time to avoid overfitting, whereas models for recurrent surgery included only the RS and no other covariates.

\section{RESULTS}

\section{Patient characteristics}

A total of 207 patients with CD had an ileocolonoscopy $\leq 12$ months after ileocolonic resection. Median age was 36 years (interquartile range, 25-48); $112(50.2 \%)$ were women. Median follow-up time was 3.9 years (interquartile range, 2.7-5.9).

Most patients had ileal or ileocolonic disease with stricturing or penetrating disease phenotype. No significant difference was observed between patients with RS i0 to i1, i2a, $\mathrm{i} 2 \mathrm{~b}$, and i3 to i4 regarding history of previous ileocolonic resection $(35 \%, 35 \%, 52 \%$, and $44 \%$, respectively; $P=.26$ ). Treatment with immunomodulatory agents and biologics was common before and after ileocolonic resection. The most common indications for surgery were ileal stricture and uncontrolled inflammation.

Baseline demographic and clinical characteristics are presented in Table 2. The RS on index colonoscopy was scored as follows: 126 patients (60.8\%) had an RS of i0 to 1, 40 (19.3\%) had i2a, $25(12.1 \%)$ had i2b, and $16(7.7 \%)$ had i3 to i4 (Table 3). The 16 patients with an $\mathrm{RS}$ of $>\mathrm{i} 2$ were excluded from further analyses. No significant difference was seen between patients with an RS of i0 to i2 in the mean Harvey Bradshaw Index at the time of colonoscopy $(P=.25)$. 


\section{Endoscopic progression}

Of 191 patients with an RS of i0 to i2, 42 patients did not have a subsequent RS available for review because of the inability to reach the anastomosis on the subsequent colonoscopy in 5 patients, colonoscopies done at other institutions in 11 patients, and 26 patients who did not undergo recommended colonoscopies during the study period. One hundred forty-nine patients had subsequent colonoscopy reports available that described the anastomosis and were included in the analysis for the primary outcome of endoscopic progression (Table 4).

Over the median follow-up period, 25 patients (16.8\%) had endoscopic progression to i3 to i4 on follow-up colonoscopy. Among those with i0 to i1 at baseline colonoscopy, $7.4 \% \mathrm{had}$ endoscopic progression to i3 to i4, as compared with $20.6 \%$ and $55.0 \%$ of those with i2a and i2b, respectively (Table 4). Kaplan-Meier and Cox regression analyses were performed for time from index endoscopy to endoscopic progression to i3 to i4 and found the hazard ratio (HR) of endoscopic progression significantly higher with i2b lesions when compared with i0 or i1 (HR, 6.22; 95\% confidence interval [CI], 2.38-16.2; $P=.0008$ ). In contrast, patients with i2a did not have significantly higher rates of endoscopic progression when compared with i0 or i1 (HR, 2.30; 95\% CI, .80-6.66; $P=.12$ ) (Fig. 1). The HR of endoscopic progression was also higher with i2b lesions when directly compared with i2a lesions (HR, $2.7 ; 95 \%$ CI, 1.01-7.23; $P=.04)$.

Cox regression analysis was performed to assess for other factors associated with endoscopic disease progression. Adjusted analyses included the following covariates: sex, race, smoking history, previous surgery, disease location and behavior, indication for surgery, surgical anastomotic technique (end-to-end, end-to-side, side-to-side), and medical treatment before and after the index colonoscopy. After adjustment, smoking history and treatment with biologics before the index ileocolonic resection were independently associated with disease progression.

The HR for current smokers relative to nonsmokers was 3.8, whereas the HR for those treated with biologics before surgery compared with those who were not treated was 3.3. After taking into account smoking history and preoperative biologic therapy, the adjusted HRs remained similar, with a significant risk of endoscopic progression for patients with $\mathrm{i} 2 \mathrm{~b}$ but not i2a lesions (Table 5).

\section{Recurrent surgery}

All 191 patients with an RS of i0 to i2 were assessed for recurrent surgeries during the follow-up period. Recurrent surgery was an infrequent event over the follow-up period, occurring in $7.3 \%$ patients (14/191). Patients with i2b lesions had a higher risk of needing recurrent surgery when compared with i0 or i1 (HR, 3.64; 95\% CI, 1.10-12.1; $P=.034$ ). Patients with i2a lesions were not found to have a significantly elevated risk for recurrent surgery (HR, 1.43; 95\% CI, .35-5.77; $P=.62)$ (Fig. 2).

\section{DISCUSSION}

In this study evaluating the differences in outcomes between an RS of i2a and i2b on the first postoperative endoscopic evaluation, we showed that the risk of endoscopic progression and 
recurrent surgery differs between patients who scored an i2a and i2b. Patients with i2b recurrence have an increased risk of endoscopic progression and recurrent surgery, whereas patients with isolated anastomotic ulcers (i2a) did not have a statistically significant increased risk of either when compared with i0 to i1.

Our study's strength lies in the relatively large patient population studied and the use of objective measures of disease recurrence, including endoscopic progression of lesions to i3 and i4 and the need for recurrent surgery. In addition, we were able to compare different subtypes of i2 with i0 to i1, because that is the clinical question at hand when attempting to decide if escalation of therapy is necessary. We also performed Cox regression analyses and controlled for covariates associated with endoscopic disease progression and found that these did not significantly change the results.

Previous studies have shown conflicting results in regard to the risk of clinical disease recurrence in patients with $\mathrm{i} 2 \mathrm{a}$ and $\mathrm{i} 2 \mathrm{~b}$ lesions. One study evaluating azathioprine for the prevention of postoperative $\mathrm{CD}$ recurrence ${ }^{9}$ reported a subgroup of 15 patients who had lesions isolated to the anastomosis (i2a) on their postoperative colonoscopy and found that none of these patients progressed to have clinical disease activity during the follow-up period. Another study evaluating the probability of clinical recurrence in patients classified as $\mathrm{i} 2 \mathrm{a}$ or $\mathrm{i} 2 \mathrm{~b}$ on their postoperative colonoscopy reported 23 patients with i2a lesions and 27 patients with i2b lesions and found no difference in the risk of clinical recurrence between these 2 groups of patients. ${ }^{11} \mathrm{~A}$ recently published study is the largest of CD patients who had undergone ileocolonic resection and had a colonoscopy within 1 year. ${ }^{12}$ This analysis of 365 patients compared clinical postoperative recurrence and need for endoscopic dilatation or new surgical intervention between patients with i2a and $i 2 b$ lesions. In this study no difference was observed in terms of clinical postoperative recurrence and need for intervention between patients with i2a or i2b lesions.

Our study included CD patients who had ileocolonoscopy $\leq 2$ months after ileocolonic resection scored as RS i0 to i2 and who had >1 year of follow-up after the endoscopic procedure. We attempted to answer the important question regarding the significance of finding $\mathrm{i} 2 \mathrm{a}$ and $\mathrm{i} 2 \mathrm{~b}$ lesions and whether there is a difference in the risk of disease relapse between these 2 subclassifications of i2 recurrence when compared with i0 to i1 using objective endpoints.

Analysis of objective outcomes are important in patients with $\mathrm{CD}$ because the correlation between symptoms and endoscopic disease activity is poor. ${ }^{13}$ This might be especially true in the postoperative $\mathrm{CD}$ patient in which a recent study showed that the type of anastomosis may drive clinical symptoms and healthcare utilization without evidence of endoscopic disease recurrence. ${ }^{14}$ Another recent study from our group found that African American race was significantly associated with clinical but not endoscopic recurrence after ileocolonic resection when compared with whites. ${ }^{15}$ Likewise, the PREVENT study, ${ }^{16}$ which evaluated the efficacy of infliximab in preventing postoperative recurrence of $\mathrm{CD}$, did not meet its primary endpoint of superiority of infliximab to placebo in preventing clinical recurrence (a composite of clinical symptoms and endoscopic recurrence). However, the study showed a significant benefit of infliximab in preventing endoscopic recurrence based on the RS, and 
indeed this study is often cited by society guidelines when recommending use of anti-tumor necrosis factor therapy for the postoperative prevention of $\mathrm{CD} ., 7$

These studies underline the importance of evaluating objective disease outcomes rather than clinical recurrence in studies of postoperative $\mathrm{CD}$, and may explain the discrepancy encountered in the results of previous investigations looking at i2a recurrence, which evaluated clinical disease relapse as the primary outcome.

The major limitation of our study is its retrospective single-center setting with the inherent risk of bias and incomplete data for some of our patients. Another limitation is the limited number of patients observed to progress to i3 to i4 $(n=25)$ and recurrent surgery $(n=14)$; there-fore, CIs surrounding our risk estimates are wide, and we cannot definitely conclude that I2a lesions do not confer increased risk of disease progression. Interobserver reproducibility of the RS has also been shown to be suboptimal. ${ }^{17}$ We reviewed all colonoscopy reports and images to reduce this risk, and discrepancies were solved by a third investigator when indicated. Furthermore, to mitigate these risks, we included all postoperative $\mathrm{CD}$ patients undergoing ileocolonoscopy within the study period and adopted objective endpoints such as endoscopic progression and recurrent surgery. Our study confirmed previously known risk factors such as smoking to be associated with the risk of endoscopic progression. Interestingly, preoperative, but not postoperative, biologic therapy was associated with a risk of endoscopic progression. This may be due to the underpowered size of the study population or the differential effects of these factors on disease recurrence. Preoperative biologic therapy might also have been a surrogate marker of patients with a more progressive and severe phenotype who might possibly be more likely to have disease relapse. To confirm the findings of this work and reduce the effects of confounders, however, a prospective study of adequate power looking at the outcomes of patients with i2a versus $\mathrm{i} 2 \mathrm{~b}$ recurrence should be performed, because these seem to represent over $30 \%$ of the RS encountered at the first postoperative colonoscopy.

Our results are significant, because prevention of post-operative $\mathrm{CD}$ is important to avoid clinical recurrence and additional intestinal resection. ${ }^{4,7}$ Patients with i2 scores are at an intermediate risk of clinical recurrence. ${ }^{6,9}$ Thus, improved subclassification of RS i2 lesions has the potential to lead to optimized therapy in high-risk individuals but not to overtreat patients at low risk of recurrence.

In this study we have added guidance to a frequently encountered clinical problem regarding the risk of objective disease progression in patients with i2 recurrence and have shown differential risks associated with $\mathrm{i} 2 \mathrm{a}$ and $\mathrm{i} 2 \mathrm{~b}$ lesions. These results suggest that a different management strategy may be applied to patients with the different subtypes of i2 recurrence, but as noted above a larger confirmatory study is needed.

In conclusion, this study of $\mathrm{CD}$ patients after ileocolonic resection suggests that $\mathrm{i} 2 \mathrm{a}$ and $\mathrm{i} 2 \mathrm{~b}$ lesions have different risks of disease progression. We recommend escalation of medical therapy in patients with $\mathrm{i} 2 \mathrm{~b}$ lesions; however, we propose that patients with lesions confined to the anastomosis (i2a) may be followed closely without treatment escalation because these 
lesions seem to have a lower risk of endoscopic disease progression. Further prospective studies are warranted to confirm our findings.

\section{Acknowledgments}

The following authors disclosed financial relationships relevant to this publication: S. R. Dalai: Grant recipient from Pfizer. A. Sakuraba: Funding recipient from AbbVie, Celltrion, and Takeda. R. D. Cohen: Consultant at Abbvie, Celgene, Janssen, Pfizer, Takeda, and UCB Pharma. D. T. Rubin: Consultant for and grant recipient from Abbvie, Merck \& Co, Janssen, Takeda, and Pfizer. J. Pekow: Grant recipient from Takeda and Abbvie; consultant for Verastem; advisory board member for Pfizer and Janssen.

\section{Abbreviations:}

$\begin{array}{ll}\text { CD } & \text { Crohn's disease } \\ \text { Cl } & \text { confidence interval } \\ \text { HR } & \text { hazard ratio } \\ \text { RS } & \text { Rutgeert score }\end{array}$

\section{REFERENCES}

1. Abraham C, Cho JH. Inflammatory bowel disease. N Engl J Med 2009;361:2066-78. [PubMed: 19923578]

2. Peyrin-Biroulet L, Loftus EV Jr, Colombel JF, et al. The natural history of adult Crohn's disease in population-based cohorts. Am J Gastroenterol 2010;105:289-97. [PubMed: 19861953]

3. Rutgeerts P, Geboes K, Vantrappen G, et al. Natural history of recurrent Crohn's disease at the ileocolonic anastomosis after curative surgery. Gut 1984;25:665-72. [PubMed: 6735250]

4. Buisson A, Chevaux JB, Allen PB, et al. Review article: the natural history of postoperative Crohn's disease recurrence. Aliment Pharmacol Ther 2012;35:625-33. [PubMed: 22313322]

5. Lichtenstein GR, Loftus EV, Isaacs KL, et al. ACG clinical guideline: management of Crohn's disease in adults. Am J Gastroenterol 2018;113: 481-517. [PubMed: 29610508]

6. Rutgeerts P, Geboes K, Vantrappen G, et al. Predictability of the postoperative course of Crohn's disease. Gastroenterology 1990;99:956-63. [PubMed: 2394349]

7. Gionchetti P, Dignass A, Danese S, et al. 3rd European evidence-based consensus on the diagnosis and management of Crohn's disease 2016: Part 2: surgical management and special situations. J Crohns Colitis 2017;11:135-49. [PubMed: 27660342]

8. Sandborn WJ, Feagan BG, Hanauer SB, et al. A review of activity indices and efficacy endpoints for clinical trials of medical therapy in adults with Crohn's disease. Gastroenterology 2002;122:51230. [PubMed: 11832465]

9. Domenech E, Manosa M, Bernal I, et al. Impact of azathioprine on the prevention of postoperative Crohn's disease recurrence: results of a prospective, observational, long-term follow-up study. Inflamm Bowel Dis 2008;14:508-13. [PubMed: 18183602]

10. Silverberg MS, Satsangi J, Ahmad T, et al. Toward an integrated clinical, molecular and serological classification of inflammatory bowel disease: report of a Working Party of the 2005 Montreal World Congress of Gastroenterology. Can J Gastroenterol 2005;19(Suppl A):5A-36A.

11. Bayart P, Duveau N, Nachury M, et al. Ileal or anastomotic location of lesions does not impact rate of postoperative recurrence in Crohn's disease patients classified i2 on the Rutgeerts score. Dig Dis Sci 2016;61:2986-92. [PubMed: 27401274]

12. Riviere P, Vermeire S, Irles-Depe M, et al. No change in determining Crohn's disease recurrence or need for endoscopic or surgical intervention with modification of Rutgeerts' scoring system. Clin Gastroenterol Hepatol 2019;17:1643-5. [PubMed: 30291910] 
13. Modigliani R, Mary JY, Simon JF, et al. Clinical, biological, and endoscopic picture of attacks of Crohn's disease. Evolution on prednisolone. Groupe d'Etude Therapeutique des Affections Inflammatoires Digestives. Gastroenterology 1990;98:811-8. [PubMed: 2179031]

14. Gajendran M, Bauer AJ, Buchholz BM, et al. Ileocecal anastomosis type significantly influences long-term functional status, quality of life, and healthcare utilization in postoperative Crohn's disease patients independent of inflammation recurrence. Am J Gastroenterol 2018;113: 576-83. [PubMed: 29610509]

15. Anyane-Yeboa A, Yamada A, Haider H, et al. A comparison of the risk of postoperative recurrence between African-American and Caucasian patients with Crohn's disease. Aliment Pharmacol Ther 2018;48:933-40. [PubMed: 30126019]

16. Regueiro M, Feagan BG, Zou B, et al. Infliximab reduces endoscopic, but not clinical, recurrence of Crohn's disease after ileocolonic resection. Gastroenterology 2016;150:1568-78. [PubMed: 26946343]

17. Marteau P, Laharie D, Colombel JF, et al. Interobserver variation study of the Rutgeerts score to assess endoscopic recurrence after surgery for Crohn's disease. J Crohns Colitis 2016;10:1001-5. [PubMed: 27068432] 
Use your mobile device to scan this QR code and watch the author interview. Download a free QR code scanner by searching "QR Scanner” in your mobile device's app store.

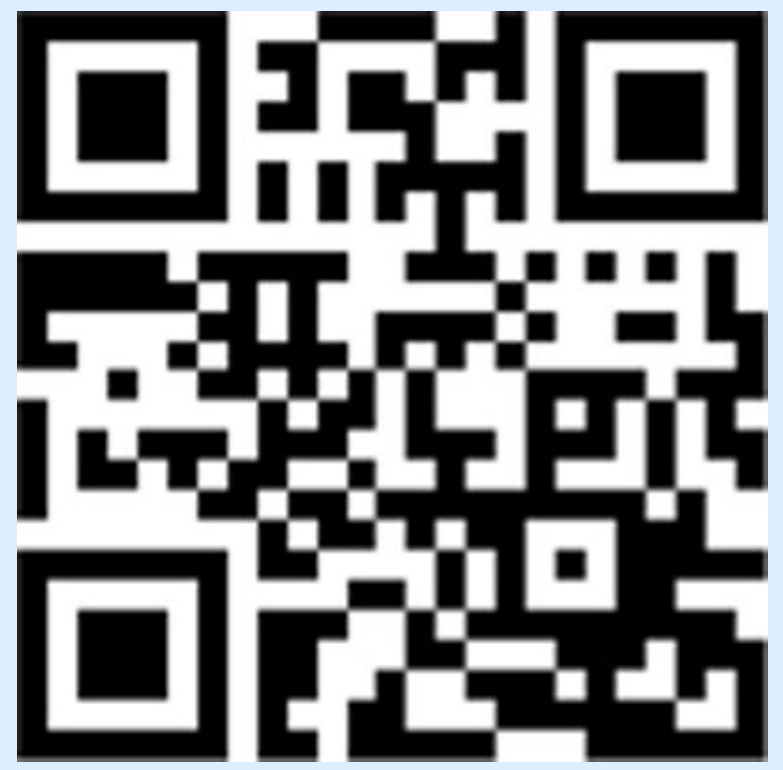

\section{GIE on LinkedIn}

Find $G I E$ on LinkedIn. Followers will receive news updates and links to author interviews, podcasts, articles, and tables of contents. Use the QR code or search on LinkedIn for "GIE: Gastrointestinal Endoscopy with Editor Michael B. Wallace" and follow us today. 


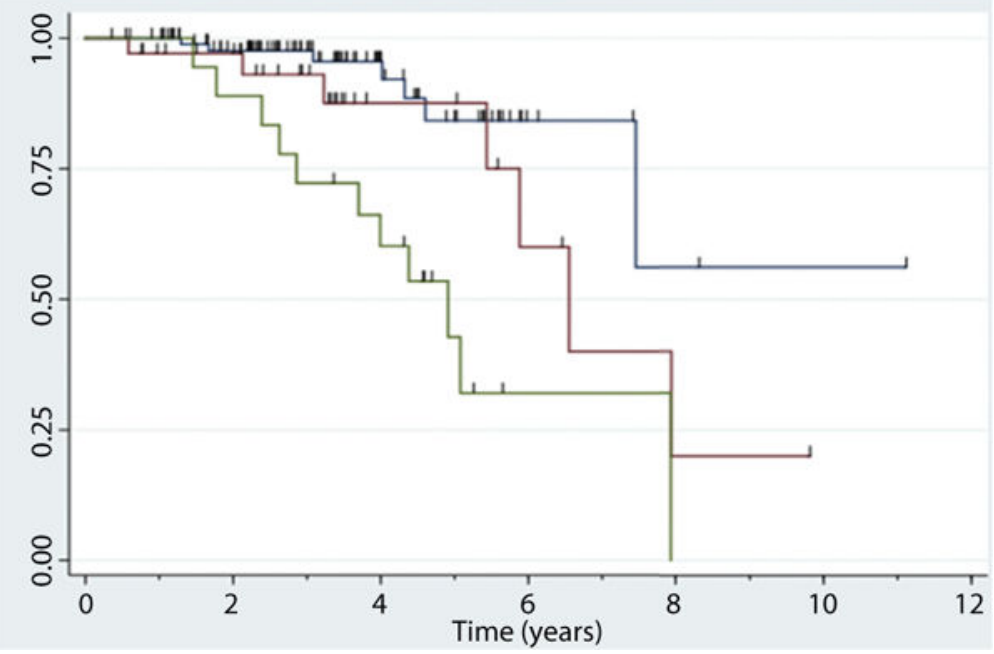

Figure 1.

Time to endoscopic progression based on Rutgeert score (RS) at the initial postoperative colonoscopy. Kaplan-Meier curves of time from first postoperative colonoscopy to RS of i3 to i4. Blue line, RS 0 to 1 ; red line, RS 2a; green line, RS $2 \mathrm{~b}$. 


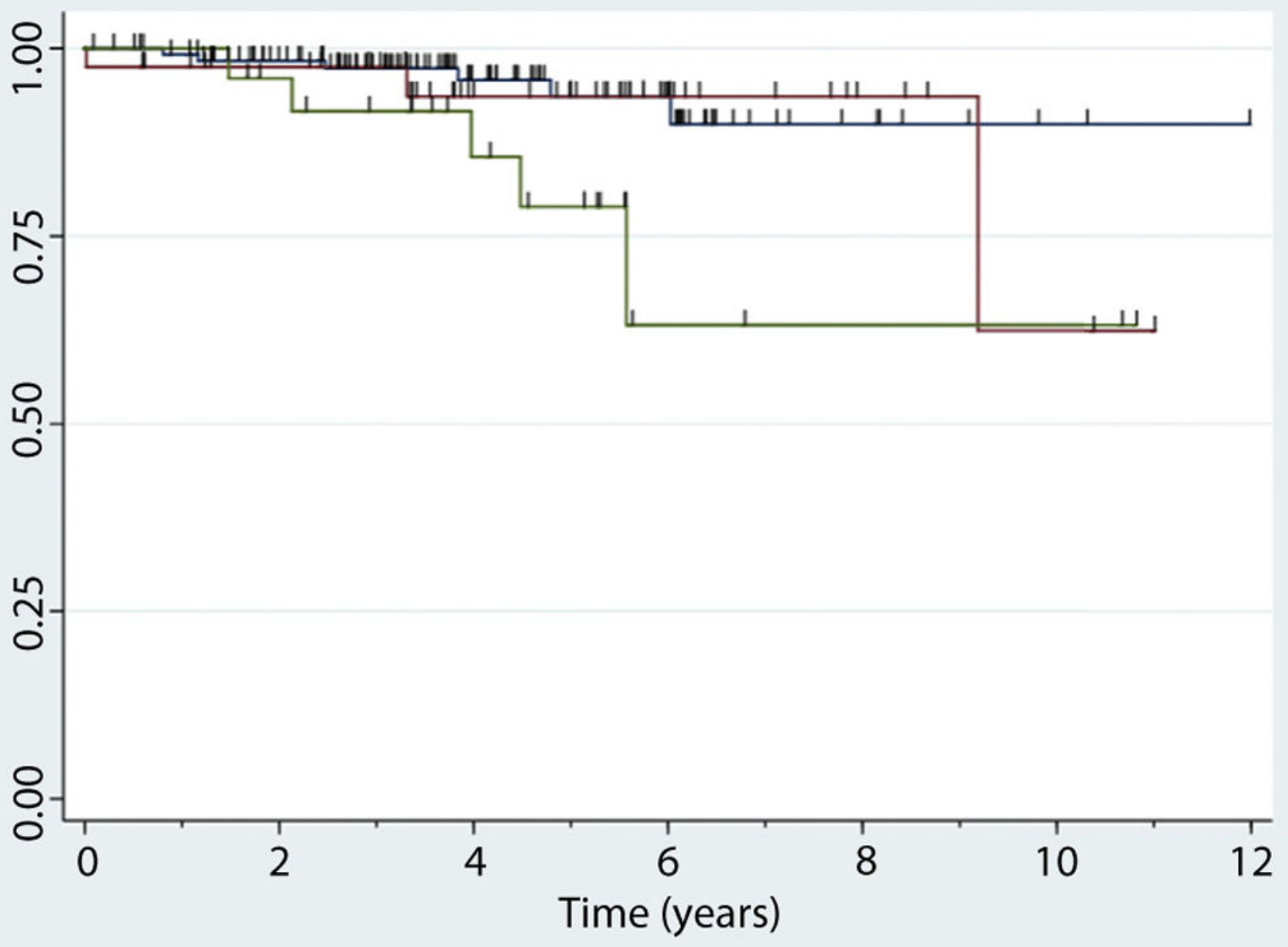

Figure 2.

Time to recurrent surgery based on Rutgeert score (RS) at the initial postoperative colonoscopy. Kaplan-Meier curves of time from first postoperative colonoscopy to recurrent surgery. Blue line, RS 0 to 1; red line, RS 2a; green line, RS 2b. 
TABLE 1.

Modified Rutgeert score

\begin{tabular}{|c|c|c|}
\hline Grade & Endoscopic finding & $\begin{array}{c}\text { Risk of } \\
\text { symptomatic } \\
\text { recurrence at } \\
5 \text { years }(\%)\end{array}$ \\
\hline i0 & No lesions ;n the distal ileum & 6 \\
\hline i1 & Fewer than 5 aphthous lesions in the distal ileum & 6 \\
\hline i2 & $\begin{array}{c}\text { More than } 5 \text { aphthous lesions with normal mucosa between the lesions or skip areas of larger lesions or lesions } \\
\text { confined to ileocolonic anastomosis }\end{array}$ & 27 \\
\hline i2a & Lesions confined to the ileocolonic anastomosis & \\
\hline $\mathrm{i} 2 \mathrm{~b}$ & $\begin{array}{c}\text { More than } 5 \text { aphthous ulcers or larger lesions with normal mucosa in between, in the neoterminal ileum (with or } \\
\text { without anastomotic lesions) }\end{array}$ & \\
\hline i3 & Diffuse aphthous iletis with diffusely inflamed mucosa & 63 \\
\hline i4 & Diffuse inflammation, with larger lesions: large ulcers and/or nodules/ cobble and/or narrowing/stenosis & 100 \\
\hline
\end{tabular}

Adapted from Rutgeerts et al. 6 
TABLE 2.

Baseline demographic and clinical characteristics $(n=207)$

\begin{tabular}{|c|c|}
\hline Characteristics & Value \\
\hline Female & $112(54.2)$ \\
\hline Median age at index colonoscopy, y (interquartile range) & $36.1(26-47.7)$ \\
\hline \multicolumn{2}{|l|}{ Smoking status } \\
\hline Current smoker & $17(12.3)$ \\
\hline Ex-smoker & $19(13.7)$ \\
\hline Never smoked & $102(73.9)$ \\
\hline \multicolumn{2}{|l|}{ Age at diagnosis } \\
\hline A1: $<16$ & $30(18.7)$ \\
\hline A2: $17-40$ & $118(73.7)$ \\
\hline A3: $>40$ & $12(7.5)$ \\
\hline \multicolumn{2}{|l|}{ Disease phenotype } \\
\hline B1: non-stricturing, non-penetrating & $15(9.5)$ \\
\hline B2: stricturing & $75(47.5)$ \\
\hline B3: penetrating & $67(42.4)$ \\
\hline \multicolumn{2}{|l|}{ Disease location } \\
\hline L1: ileal & $51(31.8)$ \\
\hline L2: colonic & $11(6.8)$ \\
\hline L3: ileocolonic & $97(60.6)$ \\
\hline Perianal disease & $51(69.8)$ \\
\hline \multicolumn{2}{|c|}{ Previous surgery (stratified by Rutgeert score at index colonoscopy) } \\
\hline i0-i1 & $44 / 126(35)$ \\
\hline $\mathrm{i} 2 \mathrm{a}$ & $14 / 40(35)$ \\
\hline $\mathrm{i} 2 \mathrm{~b}$ & $13 / 25(52)$ \\
\hline i3-i4 & $7 / 16$ (44) \\
\hline \multicolumn{2}{|l|}{ Treatment before surgery } \\
\hline Steroids & $74(45.1)$ \\
\hline
\end{tabular}




\begin{tabular}{|c|c|}
\hline Characteristics & Value \\
\hline Biologics & $59(35.9)$ \\
\hline Immunomodulator & 65 (39.6) \\
\hline Antibiotics & $35(21.3)$ \\
\hline Mesalamine & $22(15)$ \\
\hline \multicolumn{2}{|l|}{ Treatment after surgery } \\
\hline Steroids & $35(21.5)$ \\
\hline Biologics & $60(36.8)$ \\
\hline Immunomodulator & $74(45.4)$ \\
\hline Antibiotics & $41(25.1)$ \\
\hline \multicolumn{2}{|l|}{ Indication for surgery } \\
\hline Uncontrolled inflammation & $69(42.3)$ \\
\hline Perforation & $33(22.2)$ \\
\hline Stricture & $100(61)$ \\
\hline Dysplasia & $5(3.8)$ \\
\hline \multicolumn{2}{|l|}{ Type of anastomosis } \\
\hline End-to-end & $45(27.4)$ \\
\hline Side-to-side & $98(59.7)$ \\
\hline End-to-side & $21(12.8)$ \\
\hline Medan follow-up, y (interquartile range) & $3.94(2.7-5.9)$ \\
\hline
\end{tabular}

Values are $\mathrm{n}(\%)$. 
TABLE 3.

\begin{tabular}{|c|c|}
\hline \multicolumn{2}{|c|}{, } \\
\hline Score & No. of patients (\%) \\
\hline i0 & $95(45.9)$ \\
\hline i1 & $31(14.9)$ \\
\hline $\mathrm{i} 2 \mathrm{a}$ & $40(19.3)$ \\
\hline $\mathrm{i} 2 \mathrm{~b}$ & $25(12.1)$ \\
\hline i3 & $10(4.8)$ \\
\hline i4 & $6(2.9)$ \\
\hline Total & $207(100)$ \\
\hline
\end{tabular}

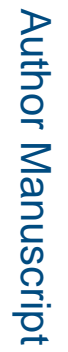

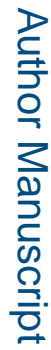

Gastrointest Endosc. Author manuscript; available in PMC 2019 October 02. 


\section{TABLE 4.}

Modified Rutgeert score of 149 patients with follow up colonoscopies included in the analysis for the primary endpoint (initial i3 and i4 excluded)

\begin{tabular}{lcc}
\hline Score & $\begin{array}{c}\text { No. of patients (\%) } \\
\text { i0/i1 }\end{array}$ & $\begin{array}{c}\text { No. of patients } \\
\text { progressed to i3/i4 (\%) }\end{array}$ \\
\hline $\mathrm{i} 2 \mathrm{a}$ & $34(22.8)$ & $7(7.4)$ \\
\hline $\mathrm{i} 2 \mathrm{~b}$ & $20(13.4)$ & $7(20.6)$ \\
\hline Total & $149(100)$ & $11(55.0)$ \\
\hline
\end{tabular}


TABLE 5.

Cox regression analyses: adjusted HRs for the covariate effects of smoking history (5a) and preoperative biologic therapy $(5 b)$

\begin{tabular}{lcc}
\hline Covariate & $\begin{array}{c}\text { Adjusted HR } \\
\text { (95\% CI) }\end{array}$ & $\boldsymbol{P}$ value \\
5a. Rutgeert score & & \\
\hline i2a vs i0 or i1 & $1.25(.35-4.41)$ & .728 \\
\hline i2b vs i0 or i1 & $3.64(1.18-11.2)$ & .024 \\
\hline Smoking history & & \\
\hline Ex vs never & $1.39(.42-4.58)$ & .59 \\
\hline Current vs never & $3.75(1.18-11.9)$ & .025 \\
\hline 5b. Rutgeert score & & \\
\hline i2a vs i0 or i1 & $1.81(.54-6.11)$ & .33 \\
\hline i2b vs i0 or i1 & $7.61(2.75-21.1)$ & .0001 \\
\hline Preoperative biologic therapy & \\
\hline Yes vs no & $3.30(1.26-8.61)$ & .015 \\
\hline
\end{tabular}

